
\title{
R Research Sousere \\ Determinants of child size at birth and associated maternal factor in Gurage zone: Application of ordinal logistic regression analysis
}

Gedif Mulat Alemayehu ( $\nabla$ gedifmulat@gmail.com )

Injibara University https://orcid.org/0000-0002-2860-9390

Ayele Gebeyehu Chernet

Wolkite University

kassahun Truha Dumga

Wolkite University

Research article

Keywords: Maternal factor, proportion odds model, size of child at birth, Infant morbidity, Infant mortality

Posted Date: July 22nd, 2019

DOI: https://doi.org/10.21203/rs.2.11819/v1

License: (c) (i) This work is licensed under a Creative Commons Attribution 4.0 International License.

Read Full License 


\section{Abstract}

Background Birth weight plays an important role in infant mortality and morbidity, child development, and future health of the child. Weight at birth is directly influenced by general level of health status of the mother. As the Gurage zone health office reports birth weight is one of the critical issues in Gugare zone that causes many babies short- term and long-term health consequences and tend to have higher mortality and morbidity. This study examined and identified the determinants of weight of children at birth in Gurage zone based on primary collected survey data by using ordinal logistic regression. Methods :The survey or the information has been collected on a total of 735,109 reproductive mothers in Gurage zone. Children with age less than 59 months have been considered on this study and from 735,109 reproductive mothers 897 were selected. Ordinal logistic regression techniques used for data analysis using maternal and socio- demographic variables as explanatory variables and size of a baby's at birth as the response variable and statistical package for social science (SPSS) version 23 was used for data analysis purpose. Result:According to our study, from the sampled children, $30.1 \%, 44.4 \%$ and $25.5 \%$ are small in size, medium in size and large in size, respectively. Maternal related variable were statistically significant like uneducated mother $(\beta=0.26, p=0.013)$, mothers who get antenatal visit care $2-3$ times $(\beta=-0.210, p=0.10)$, source of drinking water $(\beta=0.844, p=0.000)$ and malaria affected mothers $(\beta=0.344, p=0.000)$ Conclusions: children from Rural mothers, uneducated families, mothers who did not get more antenatal care visits, poor families, mothers who drink non -improved water, mothers who are affected by malaria during pregnancy, teenager mothers are small in size at birth or they are not normal at birth. Mothers' pregnancy problem and small size of children at birth are directly related.

\section{Background}

One of the poor outcomes of pregnancy that has caught the attention of the World Health Organization (WHO) is the size of children at birth which is directly related to low birth weight. A child's birth weight or size at birth is an important indicator of the child's vulnerability to the risk of childhood illnesses and the child's chances of survival. Children whose birth weight is less than 2.5 kilograms, or children reported to be "very small" or "smaller than average", have a higher than average risk of early childhood death (EDHS, 2011)

Birth weight is affected to a great extent by the mother's own fetal growth and her diet from birth to pregnancy, and thus, her body composition at conception. Mothers in deprived socio-economic conditions frequently have low birth weight infants. In those settings, the infant's low birth weight emerges primarily from the mother's poor nutrition and health over a long period of time. During pregnancy, the higher prevalence of specific and non-specific infections, or from pregnancy complications, underpinned by poverty aggravates the situation. Physically demanding work during pregnancy also contributes to poor fetal growth (Sananpanichkul $P$ et al, 2015)

Low birth weight infants are 2 times more likely to die during their first 28 days of life than normal birth weight infants (WHO and UNICEF, 2004). Low birth weight is also associated with impaired immune 
function, inhibited growth and cognitive development, high risks of developing acute diarrhea or pneumonia (Kramer, 1987). In addition, in long term developmental outcomes of low birth weight tends to have higher rates of subnormal growth, illnesses and neurodevelopment problems. Besides these a baby with abnormal weight can develop bleeding in brain, leading to learning or behavioral problems later in life. There is also evidence that LBW or its determinant factors are associated with a predisposition to higher rates of diabetes, cardiac disease and other future chronic health problems (Kramer, 1987).

Small size of children at birth is one of the critical issues in Ethiopia that causes many babies short- term and long-term health consequences and tend to have higher mortality and morbidity (Betew W, Muluneh EK, 2014). Only 5 percent of children in Ethiopia are weighed at birth. This is not surprising because the majority of births do not take place in a health facility, and children are less likely to be weighed at birth in a non-institutional setting. Among children born in the five years before the survey with a reported birth weight, 11 percent weighed less than 2.5 kilograms. Every single day, Ethiopia loses thousands of underfive year old children because of abnormal weight (CSA and ORC, 2011).

As the Gurage zone health officer report indicate the weight about quarter $(25 \%)$ of the new born children in Gurage zone is below average or smaller than average, this small weight exposes children to extremely high rates of morbidity and mortality. Thus this study intended to identify the risk factor for small size of children at birth.

\section{Methods}

\section{Study design and sample size}

The study has been conducted in Gurage zone which is found in south nation, nationalities and peoples' region (SNNPR), Ethiopia. According to Gurage zone health care office the total population in this zone is estimated to be 1,609,908 among this 735,109 are women in reproductive age (15-49), so the target population for this study is women in reproductive age $(735,109)$. The study conducted in Gurage zone women who have children less than five years from September to February, 2017.

Stratified, multi-stage sampling technique was employed to include study participants in to the research. The study area was first stratified in to urban and rural places since residency is known to affect the prevalence of size of children at birth.

The total 15 woredas of the study area were stratified in to two strata, urban and rural, each containing 2 town administrates and 13 woredas, respectively.

Then in the first stage, one town administrative (butajira), two rural woredas (Abeshige and Gumer) were selected proportionally-based on the number of woredas in each stratum- through

lottery method. In the second sampling stage, simple random sampling technique was used to take households from each of the selected three woredas-by taking into account the number of households in 
each of the sampled woreda-until the calculated sample size in the respective woreda was reached to achieve the sample size of 897 households in total.

\section{Data collection procedure}

The data for this study were collected by organized questionnaire recorded birth weight, if available from written records or mother's recall, for all births in the five years preceding the study. Birth weight may not be known for many babies, and particularly for babies delivered at home and not weighed at birth, the mother's estimate of the baby's size at birth was also obtained.

\section{Study variables}

This study tried to include the most important and expected determinants of child size at birth from various literatures. The explanatory variables at individual and household levels included were Place of residence, Mothers education level, Wealth index of family, Family size, Frequency of antenatal visit during pregnancy, Sex of child, Birth order, Previous birth interval, Vaccination during pregnancy, Abortion status of mothers, Age at first birth, Frq. of listening to radio, Malaria history of mothers, Source of drinking water, and use of contraceptive

\section{Method of data analysis}

Ordinal Logistic regression was used to analysis the data in this study because the size of child at birth ordered. Specifically, proportional odds model (POM) was employed.

\section{Results}

From the sampled children, $30.1 \%, 44.4 \%$ and $25.5 \%$ are small in size, medium in size and large in size, respectively. The distribution of small size of baby at birth or low birth weight by key characteristics of the child, mother, and household among children whose mothers were interviewed is shown in Table 1 (see Supplementary Files).

\section{Determinants of size of children at birth}

The result of uni-variable ordinal logistic regression analysis indicated that the variables sex $(\mathrm{p}$-value $=$ $0.312)$, abortion history ( $p$-value $=0.136)$, economic source of family, and frequency of watching TV( $p$ value $=0.445$ ), were not significant at $5 \%$ Ivel of significant. Hence, the final multivariable model excludes insignificant variables from the analysis. Accordingly, the deviance-based chi-square test provided a chisquare value of $360.582(p=.000)$ for the finale Model which would imply that the model is good fit as compared to the intercept only model. Further more, the Chi-square test statistic is not significant (). Therefore, there is no enough evidence to reject the null hypothesis for the final model. Thus, the 
proportional odds assumption appears to have held for the final model. (see Table 2 in the Supplementary Files)

\section{Discussion}

When the proportional odds model is used in the analysis of ordinal data, the coefficients of the explanatory variables in the model are interpreted as the logarithm of the ratio of the odds of the response variable. This means that estimates of this odds ratio, and corresponding confidence intervals can be easily found from the fitted model. The discussion and interpretation of the parameters corresponding to the variables which are found significant in the final model as shown in table 2 are described in the following section.

The result indicates that place of residence is a significant covariate. The estimated odds ratio $(\exp ()=\exp (-.13)=0.878)$ indicates that urban children are 0.878 times less likely to be small child at birth as compared to rural children holding all other variables constant. The odds ratio could be as low as 0.9166 and as high as 1.4181 with $95 \%$ confidence. The study is consistent with Mwabu (2008) that birth weights are lower in rural than in urban areas.

From Table 2 we can also observe that birth order is significantly related with the size of children at birth. As compared to 3 and above order children the first order child being small at birth is 0.56 times less likely making that all variables constant.as mothers get older they get small size child at birth. The result is inconsistent with Magadi et al. (2004) which state that birth order as an important factor influencing birth weight and first order births are on average more likely to be small babies than higher order births. The study is also inconsistent with Mwabu (2008) that states birth weight is positively associated with higher birth orders, with the first born child being significantly lighter than subsequent children.

Mothers' education level is a significant predictor of size of child at birth. the estimated odds ratio $(O R=1.29)$ implies that children from un educated mothers' are 1.29 times more likely to be small size at birth as compared to child from mothers' with education level is secondary and above(reference category) keeping all other covariates constant. This number can go up 1.568 and can down to 0.996 with $95 \%$ confidence. This study result is consistent with Tuntiseranee et al. (1999) that maternal education is a significant factor increasing the risk to deliver LBW baby even after adjustment for possible confounding factors such as maternal age, parity, obstetrical anamnesis and pre-natal care level. The study is also consistent with Siza (2008) and Khatun and Rahman (2008) that there is a linear decrease in low birth weights of newborns as maternal educational level increased and maternal education level play an important role in the incidence of low birth weight. Another study also found mother education is significant for low birth weight (Hirve, Ganatra, 1994). They indicated that education improves the ability of mothers to implement simple health knowledge and facilitates their capacity to manipulate their environment including health care facilities, interact more effectively with health professionals, obey with treatment recommendations, and keep their environment clean. Furthermore, 
educated women have adequate nutritional status and antenatal care visit during pregnancy for child birth safety.

In our study antenatal visit care (number of times pregnant women get antenatal care) was found statistically significant variable associated with size of child at birth. The odds ratio for pregnant women who get antenatal visit 2-3 times is $(\mathrm{OR}=0.77)$ implies that children from mothers who get antenatal visit care 2-3 times during pregnancy period are 0.77 times less likely to be small size at birth as compared to child from mothers who do not get any antenatal visit care keeping all other covariates constant. Mothers who get complete antenatal visit care can give normal child. The finding is consistent with Magadi et al. (2000) that early antenatal care initiation also associated with heavier birth weights and with Khatun and Rahman (2008) that number of antenatal care visit attended $(p<0.001,0 R=29.386)$ play an important role in the incidence of low birth weight.

The model results portrayed that source of drinking water is a significant variable. Children from households with non-improved source of drinking water are not normal in size during birth. Children from mothers who drink river water are 1.65 times more likely to be small size at birth as compared to children from mothers who drink piped water $(\mathrm{p}<.027$ and $\mathrm{OR}=1.65)$. And children from mothers who drink from hole water are 2.32 times more likely to be small size at birth as compared to children from mothers who drink piped water $(\mathrm{p}<.000$ and $\mathrm{OR}=2.32)$ when other factors are constant. The study agree with Dharma lingam et al. (2010) conducted a study in India using national survey data investigated the association between the mother's nutritional status and birth weight of her newborn. They found that safe drinking water was important determinant for size of children at birth. Pure sanitation conditions are linked with size of children at birth, even if indirectly, since such conditions are associated with greater numbers of infectious and parasitic diseases, which in turn contributed towards diminishing the health status of baby.

The model result also showed that wealth index (income of the household) is a significant predictor for size of children at birth. The estimated odds ratio for high wealth index and medium wealth index (OR= $0.60 \& p<.000,0 R=0.733 \& P<.012$ ) indicate that children from rich families are 0.60 times less likely to be small size at birth and children from medium income families are 0.733 times less likely to small/medium size at birth as compared to children from poor families keeping other variables constant. The result of this study agrees with Ipadeola et al. (2013) that wealth index is positively associated with child's weight at birth.

The health condition of pregnant mother has statistically significant effect on the size of children at birth. Pregnant mothers who are affected by malaria bring forth small child. Children born from malaria affected mothers are 1.41 times more likely to be small size at birth than children born from healthy mothers. The result is consistent with Siza (2008) which stats that malaria (14.8\%) contributed to high prevalence of low birth weight. The study agree with Kramer, (1998) that the maternal environment is the most important determinant of birth weight and factors such as malaria that prevent normal circulation 
across the placenta cause shortage of nutrient and oxygen supply to the fetus and restricts the growth of the fetus.

The model results also showed that the age of mothers at birth is a significant predictor of size of children at birth. The estimated odds ratio for mothers of age 21 year and above years $(O R=0.37 \&$ $\mathrm{P}<0.000$ ) indicates that children born from mothers whose age at birth is 21 year and above are 0.37 times less likely to be small size at birth as compared to children from mother age bellow 16 years keeping other variables constant. The odds ratio could be as low as 0.294 and as high as 0.473 with $95 \%$ confidence. The study is consistent with EDHS, (2011) that Low birth weight is more common among children of the youngest mothers, age less than 20 (13 percent) and older mothers, age 35-49 (17 percent). Children born to very young mothers (<20 years) were the most likely to be reported as small. The study also agreed with Ipadeolaet al. (2013) that Age of mother at the birth of a child has also been shown to be of risk to pregnancy outcomes. Teenage mothers were more likely to give birth to children with low birth weight. Children from mothers in the age range 25 to 39 years were about 1.26 times more likely to weigh more at birth compared with children from teenage mothers.

\section{Conclusion}

According to the study, place of residence, mother's level of education, birth order of the child, source of drinking water, abortion history of mother, frequency of antenatal care visit, vaccination during pregnancy, frequency listening to radio, malaria history of mothers, age of mothers at birth and previous birth interval of child are all important in reducing the incidence of small size at birth. children from Rural mothers, uneducated families, mothers who did not get more antenatal care visits, poor families, mothers who drink non -improved water, mothers who are affected by malaria during pregnancy, teenager mothers are small size at birth or they are not normal at birth. Size of children at birth and mothers pregnancy problem is directly related on this study.

\section{Abbreviations}

AIC Akakie information criteria

BIC Basian information criteria

$\mathrm{Cl} \quad$ Confidence Interval

CSA Central Statistical Agency

EDHS Ethiopia Demographic and Health Survey

GOF Goodness of fit test

LBW Low birth weight 
LL

Logit

POM

PPOM

SNNP

SPSS

UNICEF

WHO
Log Likelihood

Log of Odds

Proportional Odds Model

Partial Proportional Odds Model

Southern Nations, Nationalities, and People's

Statistical Package for Social Science

United Nations Children's Fund

World Health Organization

\section{Declarations}

\section{Acknowledgment}

Our gratitude goes to Wolkite university for the permission of budget to do this paper, secondly we thank very much Butajira town administrative, Gumer woreda and Abeshige woreda health office for their great contribution to get basic information regard to size of children at birth.

Finally we would like to thank data collectors who are supporting us by collecting basic information from respondents even in difficult area of Gurage zone.

\section{Funding}

We did not receive external funding for this research.

\section{Availability of data materials}

The author couldn't attach the data because of unfortunately the data were lost after the study has finished.

\section{Authors' contributions}

1. designed the study, performed the statistical analysis, prepared the manuscript, AG.collected the data and interpreted the results. KT. collected the data and entered the data. The authors also read and approved the final manuscript.

\section{Author's information}

The author currently works at Injibara University, Injibara, Ethiopia. He serve as a Lecturer and Researcher in statistics Department. Gedif obtained both Master of Science in Biostatistics and Bachelor of Science 
in Statistics from University of Gondar, Ethiopia.

\section{Competing interest}

The authors declare that they have no competing interest.

\section{Consent for publication}

This manuscript does not contain any individual person's data

\section{Ethics approval and consent to participate}

The consent to participate was verbal because the study was conducted by using verbal questionnaires from care giver or mothers. Informed verbal consent was obtained from each study subject. Each respondent was informed about the objective of the study and assurance of confidentiality. The survey was commenced after obtaining permission from Gurage Zonal Health office and Wolkite University Research Ethics Review Committee, the committee approved the ethical clearance and consent to participate.

\section{References}

1. Andersson SW, Niklasson A, Lapidus L, Hallberg L, Bengtsson C, Hulthén L. Sociodemographic characteristics influencing birth outcome in Sweden, 1908-1930. Birth variables in the Population Study of Women in Gothenburg. Journal of Epidemiology \& Community Health. 2000 Apr 1;54(4):269-78.

2. Ashworth A. Effects of intrauterine growth retardation on mortality and morbidity in infants and young children. European journal of clinical nutrition. 1998 Jan;52:S34-41.

3. Akaike, Hirotugu. A new look at the statistical model identification. IEEE transactions on automatic control 19.6 1974: 716-723.

4. Central Statistical Agency, ORC Macro 2006, and Ethiopia Demographic and Health Survey 2011: Addis Ababa, Ethiopia, and Calverton, Maryland, USA: Central Statistical Agency and ORC Macro.

5. Ebomoyi E, Adetoro OO, Wickremasinghe AR. Birthweight and sociobiological factors in llorin, Nigeria. Journal of biosocial science. 1991 Oct;23 (4):417-23.

6. Ethiopian Demographic and Health Survey 2011. Central Statistics Agency (CSA), Addis Ababa, Ethiopia.

7. Hirve SS, Ganatra BR. Determinants of low birth weight: a community based prospective cohort study. Indian pediatrics. 1994 Oct 1;31(10):1221-5.

8. Khatun S, Rahman M. Socio-economic determinants of low birth weight in Bangladesh: a multivariate approach. Bangladesh Medical Research Council Bulletin. 2008;34(3):81-6.

9. Kramer MS. Determinants of low birth weight: methodological assessment and meta-analysis. Bulletin of the World Health Organization. 1987;65(5):663. 
10. Hosmer DW, Lemeshow S. Applied Logistic Regression. John Wiley \& Sons. New York. 2000.

11. Magadi M, Diamond I, Madise N, Smith P. Pathways of the determinants of unfavourable birth outcomes in Kenya. Journal of Biosocial Science. 2004 Mar;36(2):153-76.

12. Magadi MA, Diamond I, Madise N. Individual and community level factors associated with premature births, size of baby at birth and caesarean section deliveries in Kenya. African Population \& Health Research Center; 2000 January: 34(1): 123-34.

13. McCormick MC. The contribution of low birth weight to infant mortality and childhood morbidity. New England journal of medicine. 1985 Jan 10;312(2):82-90.

14. Mwabu G. The production of child health in Kenya: a structural model of birth weight. Journal of African Economies. 2008 Jul 22;18(2):212-60.

15. Chuku SN. Low birth weight in Nigeria: Does antenatal care matter. Journal of Arts in Development Studies, Institute of Social Study Netherland. 2008 Nov;12:66-71.

16. Dickute J, Padaiga Z, Grabauskas V, Gaizauskiene A, Basys V, Obelenis V. Do maternal social factors, health behavior and work conditions during pregnancy increase the risk of low birth weight in Lithuania?. Medicina (Kaunas, Lithuania). 2002;38(3):321-32.

17. Padaiga Z, Basys V, Gaizauskiene A. Social inequalities in newborns health in Lithuania during 1995-1997. Lietuvos akušerija ir ginekologija. 1999;2:304-9.

18. Rehan NE, Tafida DS. Low birthweight in Hausa infants. Nigerian journal of paediatrics. 1981;8(2):35-9.

19. Siza JE. Risk factors associated with low birth weight of neonates among pregnant women attending a referral hospital in northern Tanzania. Tanzania journal of health research. 2008;10(1):18.

20. Tuntiseranee P, Olsen J, Chongsuvivatwong V, Limbutara S. Socioeconomic and work related determinants of pregnancy outcome in southern Thailand. Journal of Epidemiology \& Community Health. 1999 Oct 1;53(10):624-9.

21. Van Ginneken JK, Muller AS. Maternal and child health in rural Kenya: an epidemiological study.

22. World Health Organization, Unicef. Low birthweight: country, regional and global estimates.

23. Betew W, Muluneh EK. Determinants of Low Birth Weight among Children Aged 0 to 59 Months in Ethiopia. International Journal of Pure and Applied Sciences and Technology. 2014 Nov 1;25(1):14.

24. Dharmalingam A, Navaneetham K, Krishnakumar CS. Nutritional status of mothers and low birth weight in India. Maternal and child health journal. 2010 Mar 1;14(2):290-8.

25. Ipadeola OB, Samson B, Adebayo SB, Anyanti J, Jolayemi ET. Poverty levels and maternal nutritional status as determinants of weight at birth: an ordinal logistic regression approach. International Journal of Statistics and Applications. 2013;3(3):50-8.

26. Sananpanichkul P, Rujirabanjerd S. Association between maternal body mass index and weight gain with low birth weight in eastern Thailand. Southeast Asian Journal of Tropical Medicine and Public Health. 2015 Nov 1;46(6):1085. 


\section{Tables}

Due to technical limitations, tables 1 and 2 are only available as a download in the supplemental files section.

\section{Supplementary Files}

This is a list of supplementary files associated with this preprint. Click to download.

- Table2.png

- Table1.png 